\title{
NANOPARTICLE FORMULATION OF BIOFLAVONOIDS FOR ENHANCED ANTI-CANCER ACTIVITY
}

\author{
S. SATHESH KUMAR ${ }^{1}$, P. SHANMUGASUNDARAM ${ }^{2}$, M. KOMALA ${ }^{1}$, B. BHARGAVI ${ }^{1}$, J. PADMAVATHY ${ }^{*}$
}

1Department of Pharmaceutics, School of Pharmaceutical Sciences, Vels Institute of Science, Technology and Advanced Sciences (VISTAS), Chennai 600117, Tamil Nadu, India, ${ }^{2}$ Department of Pharmaceutical Chemistry and Analysis, School of Pharmaceutical Sciences, Vels Institute of Science, Technology and Advanced Sciences (VISTAS), Chennai 600117, Tamil Nadu, India

Email: padmaviji82@gmail.com

Received: 23 May 2020, Revised and Accepted: 09 Jul 2020

\begin{abstract}
Among the natural sources, plant origin drugs constitute around $25 \%$ which includes various secondary metabolites such as bioflavonoids, alkaloids, terpenes, saponins, glucosides, and lignans. The bioflavonoids belonging to the polyphenol group shows many beneficial effects like hepatoprotective, antioxidant, antibacterial, anticancer, anti-inflammatory and antiviral. The main objective of this article is to collectively present the research data published worldwide about the anticancer activity of bioflavonoids by loading them in novel formulations. Thus, the present review explored the novel formulations of the bioflavonoids with improved pharmacokinetic properties along with the enhanced anticancer activity. The major drawback with bioflavonoids is its poor solubility and bioavailability, which restricts the usage of bioflavonoids in the treatment of cancer in the market worldwide. Novel drug delivery system seems to possess many benefits like site-specific drug delivery along with minimal side effects and improves pharmaceutical and therapeutic properties of drugs compared to a conventional dosage form of bioflavonoids. The scope for improvement of anticancer activity of bioflavonoids by incorporating in novel pharmaceutical formulations like nanoparticles is very high, and it has to be considered as a potential area of research.
\end{abstract}

Keywords: Anticancer, Bioflavonoids, Novel drug delivery system, Nanoformulations

(C) 2020 The Authors. Published by Innovare Academic Sciences Pvt Ltd. This is an open access article under the CC BY license (http://creativecommons.org/licenses/by/4.0/) DOI: http://dx.doi.org/10.22159/ijap.2020v12i5.38425. Journal homepage: https://innovareacademics.in/journals/index.php/ijap

\section{INTRODUCTION}

The 'nano' prefix has been gradually applied to various areas of information over the last decade. Nanoscience, nanotechnology, nanomaterials or nanochemistry are only a few of the latest nanocontaining terms that often appear in scientific journals, in mainstream books as well as in newspapers and have become widely familiar among the general public, even among non-experts [1]. Nano derives its name from the Greek word Vavo and in Latin nanus, which means dwarf and, by extension, very small. Nanotechnology is considered to be one of the major branches of physical and biological sciences that apply nanostructures and nanophases in different scientific fields, in particular, nanomedicine and nano-based drug delivery systems [2]. 'Nanomaterials can be characterised as a material with sizes ranging from 1 to $100 \mathrm{~nm}$, which effects the frontiers of nanomedicine starting from biosensors, microfluidics, drug delivery and microarray testing to tissue engineering'. Medicine based on nanoparticle has been developed by combining treatment and imaging modalities for the diagnosis of diseases. Lipid systems such as liposomes and micelleswhich are now approved by the FDA-are the very first generation of therapy based on nanoparticles [3]. Nano drug delivery systems exhibit absorptive endocytosis take-up mechanisms and hence show greater oral bioavailability [4].

We searched literature and guidelines in Pubmed, Web of Science, Google Scholar, Scopus, CNKI and Embase databases up to 2019. The following search terms alone or matched with the Boolean operators 'AND' or 'OR' were used: "Nanoparticles", "Anticancer treatment", 'Bioflavonoids', 'Plant origin drugs', 'Nano formulations', 'Cancer' and 'Novel drug delivery systems'. We focused on full-text articles, but abstracts were considered if relevant.

Fundamentals of nanotechnology-based techniques in designing of drug

'Nanomedicine is the branch of medicine that uses the science of nanotechnology for the prevention and treatment of different diseases utilising the nanoscale materials, like biocompatible nanoparticles and nanorobots for various applications including, diagnosis, delivery, sensory or actuation purposes in a living organism' [5]. Drugs with very low solubility possess various biopharmaceutical delivery issues including limited bioaccessibility after intake through the mouth, less diffusion capacity into the outer membrane, require more quantity for intravenous intake and unwanted after-effects preceding conventional formulated vaccination process [6]. However, the utilisation of nanotechnology approaches in the mechanism of drug delivery could overwhelm all of these confinements [7]. 'This can lead to the enhancement and development of convenient routes of administration, lower toxicity, fewer side effects, improved biodistribution and extended drug life cycle. The novel drug delivery systems are mainly intended to control the release of drugs to a specific site and also target to disease site.' [8]. Their formation involves self-assembly where the building blocks are spontaneously formed in well-defined structures or patterns. In addition, barriers such as 'opsonisation/sequestration' by the mononuclear phagocyte system need to be overcome [9].

Nanostructures deliver drugs in two ways: (a) passive and (b) selfdelivery. (a) In the former, 'drugs are incorporated mainly by the hydrophobic effect in the inner cavity of the structure. Due to the low content of the drugs encapsulated in a hydrophobic environment, the expected amount of the drug is released when the nanostructure materials are targeted to a specific site' [10]. (b) Conversely, in the latter, 'the drugs to be released are conjugated directly to the "carrier nanostructure material" for easy delivery' [11]. 'In this approach, the timing of release is crucial because the drug will not reach the target site and it dissociates from the carrier very quickly, and conversely, its bioactivity and efficacy will be decreased if it is released from its nanocarrier system at the right time' [12].

'Targeting of medicine is another significant aspect that uses nanomaterials or nanoformulations as the drug delivery systems is classified into active and passive. In active targeting, moieties, such as antibodies and peptides, are combined with drug delivery system to anchor them to the receptor structures expressed at the target site' [13]. 'The prepared drug carrier complex, in passive targeting, circulates through the bloodstream and is driven to the target site by 
affinity or binding influenced by properties such as $\mathrm{pH}$, temperature, molecular site and shape' [14]. Nano, drug delivery system, is intended mainly to target and cure a disease at a particular site like cancerous tumors etc [15].

\section{Cancer}

Cancer is one of the world's most debilitating and life-aggressive diseases that causes cruel deaths. This is also the principal cause of death in urbanized countries following cardiovascular diseases [16]. About 30 percent of death from cancer was directly linked to dietary patterns. This is due to changes in diet and sedentary lifestyle adaptation, and the burden of this disease is increasing gradually every day [17].

\section{What is cancer?}

'Cancer cells do not die at a natural point in the life cycle of a cell. Cancer is a very broad term. It describes the disease that results when cellular changes cause uncontrolled cell growth and cell division'.

Many cancers cause cells to develop rapidly, while others cause cells to grow and divide more slowly. Certain types of cancer lead to noticeable tumour growth, while others, for example, leukaemia, do not. Many cells inside the body have specific functions and defined lifespan. Cell death is a part of a process called apoptosis which is normal and beneficial. A cell is instructed to die so that the body can replace it with a newer cell which works better. Cancer cells don't have the components which instruct them to stop dividing and die.

Therefore, they grow inside the body, utilising oxygen and nutrients that might regularly feed other cells. Cancerous cells can form tumours, damage the immune system and cause different changes that prevent the body from functioning regularly. Within one area of cancer, cells arise and spread through the lymph nodes. These clusters of immune cells are located throughout the body.

\section{Causes}

The causes of cancer are numerous.

The major preventable risk factors for cancer include Smoking, heavy alcohol intake, excess weight, physical inactivity, inadequate diet, etc.

Many cancer causes are not preventable. Age is currently the most significant unpreventable risk factor.

\section{Genetic factors}

Genetic factors may lead to the development of cancer. Genetic code of a person informs their cells when to divide and expire. Gene modifications can lead to erroneous instructions, which can lead to cancer.

Genes also affect protein production in the cells, which proteins contain many of the instructions for cell growth and division. Some genes change proteins that would usually repair damaged cells. It can lead to cancer. If a parent has these genes, they may pass on the altered instructions to their offspring. Some genetic changes happen after birth, and risk may be increased by factors such as smoking and exposure to sunlight.

Many modifications that may cause cancer to exist in the chemical signals that determine how the body deploys, or 'expresses' specific genes. Finally, an individual may inherit a predisposition to a form of cancer. This is usually referred to as an inherited condition of cancer. Hereditary cancer and inherited genetic mutations seems to contribute significantly upto 5-10 percent development of cancer cases. to the 5-10 percent development of cancer cases.

Types

The most common type of cancer is breast cancer, followed by lung and prostate cancers, according to the National Cancer Institute, which excluded nonmelanoma skin cancers. Smoking increases the risk of many different types of cancers.
Every year, over 40,000 people around the country are diagnosed with one of the following types of cancer: bladder, colon, rectal, renal, liver, endometrial, melanoma, leukaemia, pancreatic, thyroid and non hodgkin's.

Other types are less frequent.

\section{Cancer development and cell division}

Cancer can be classified by (i) the location of cancer in the body; and (ii) the type of tissues it forms in the body.

For example, sarcomas develop in bones or soft tissues, while carcinomas form in cells that cover the body's internal or external surfaces. Basal cell carcinomas develop in the skin, while adenocarcinomas can form in the breast. When cancer cells spread to other areas of the body, it is medically termed as metastasis.

Chemotherapy is currently the most widely used cancer medication that involves alkylating agents, antimetabolites, antitumor antibiotics, platinum analogs, all of which can potentially contribute to skin or lung cancer and adverse side effects to their long-term use in therapy. There is, therefore a need to identify new molecules for the treatment of low-cost cancer, high-efficiency cures, and fewer side effects [18].

\section{Bioflavanoids}

Bioflavonoids are antioxidants found naturally in vitamins $\mathrm{C}$ and $\mathrm{E}$ and carotenoids. These compounds protect our body cells against free radical damage. Free radicals are toxins in the body that can damage healthy cells. When this happens, it is called oxidative stress.

Many antioxidants, like flavonoids, may not be found in high concentrations in the bloodstream alone. But they may affect the transport or activity of more potent antioxidants, like vitamin $\mathrm{C}$, throughout the body. In fact, some supplements you'll find at the store contain both vitamin $\mathrm{C}$ and flavonoids together for this reason.

\section{Antioxidant power}

Researchers share that bioflavonoids may help with several health issues. They have the potential to be used therapeutically or protectively. Flavonoids may also influence the ability of vitamin C to be absorbed and utilised by the body. The antioxidant power of flavonoids is well documented in different studies. In one overview, researchers explain that antioxidants like flavonoids work in a variety of ways. They can:

- Interfere with the enzymes that create free radicals, which suppresses reactive oxygen species (ROS) formation

- Scavenge free radicals, meaning they deactivate these harmful molecules before they cause damage

- Protect and even increase antioxidant defences in the body [19]

When antioxidants stop free radicals in their tracks, cancer, ageing, and other diseases may be either slowed or prevented [20-24].

\section{Allergy-fighting potential}

Allergic diseases may respond well to taking in more bioflavonoids. This includes:

- Atopic Dermatitis

- Allergic Rhinitis

- Allergic Asthma

The Development of allergic disorders is usually associated with excess oxidative stress on the body. Flavonoids may help to scavenge free radicals and stabilize the reactive oxygen species. It could lead to fewer allergic reactions. They may also reduce inflammatory responses that contribute to diseases like asthma. 
So far, the research has suggested that flavonoids-along with improved diet habits-show potential for fighting allergic diseases. However, the mechanism of action and effectiveness of these bioflavonoids is still under research.

\section{Uses}

The flavonoids orientin and vicenin may help the body repair after an injury from radiation. The subjects in this study were mice. The mice were exposed to radiation and later given a mixture containing the bioflavonoids. In the end, the bioflavonoids proved to be efficient at scavenging the free radicals produced by radiation. They also were associated with faster DNA repair in cells that had been damaged.

Flavonoids and detoxification is another subject being explored in the research community. Some even believe that flavonoids may help clear the body toxins that lead to cancer. Studies on animals and isolated cells support these claims. Unfortunately, those on humans have not consistently shown that flavonoids do much to reduce cancer risk. Flavonoids potentially have a role in lowering one's risk for cancer, including breast and lung cancers [25-30].

Finally, bioflavonoids may have antimicrobial properties as well. In plants, they have been shown to help fight microbial infection against different microorganisms. In particular, bioflavonoids like apigenin, flavone, and isoflavones have been shown to have potent antibacterial properties.

\section{Dose}

200-250 mg of bioflavonoids each day.

\section{Food sources}

Tea, berries, onion, vegetables, flowers, leaves, mangoes, nuts grapes, soybeans, pears, apples, cucumber, cherries, carrots, pepper, citrus fruits, clove and so on.

\section{Types of bioflavonoids}

Bioflavonoids are divided into five subcategories.

- Flavonols (quercetin, kaempferol, myricetin, and fisetin)

- Flavan-3-ols (catechin, epicatech

- In gallate, gallocatechin, and theoflavin)

- Flavones (apigenin and luteolin)

- Flavonones (hesperetin, naringenin, and eriodictyol)

- Anthocyanidins (cyanidin, delphinidin, malvidin, pelargonidin, peonidin, and petunidin)

\section{Nanoparticle formulation of bioflavonoids}

\section{Quercetin}

Quercetin, a dietary flavonoid is considered to be the pillar for many of the other flavonoids. It is found to be distributed naturally in many of the diet foods such as tea, berries, apple, onion, vegetables and nuts, seeds, barks, leaves, and flowers. It is chemically $3,5,7,3$ ', 4' pentahydroxy flavones and it is found to show many pharmacological properties such as cardioprotective effect [31], antioxidant activity, analgesic activity, antiviral activity, antidiabetic activity, anticancer activity, immunosuppressor activity, antithyroid activity, and so on. The anticancer activity was shown to be exhibited by various mechanisms such as abolishing the free radicals, reaction with the estrogen receptors, proteins and transcription factors, retarding cell proliferation, initiation of apoptosis, and reducing metalloproteinase 2 and 9 expressions. Quercetin, although, has many of the therapeutic properties its use is limited due to many of the drawbacks such as less bioavailability and poor water solubility and so to improve its physicochemical properties and also to improve its targeting property the novel drug delivery systems such as nanoparticles, niosomes, and liposomes can be developed for quercetin [32].
Saha et al. [33] formulated and evaluated the nanoparticles of polylactic co-glycolic acid with hydrophobic anticancer drugs of anthracycline origin such as paclitaxel, doxorubicin, and bioflavonoid quercetin, chemoprevention by single emulsion solvent evaporation method. The surface modification of the bioflavonoid nanoparticles was carried out using the polymers of biological origin such as histones or bovine serum albumin. The formulated nanoparticles were evaluated for their physicochemical properties such as particle size analysis, zeta potential, entrapment efficiency, drug loading capacity, Fourier-transform infrared, differential scanning calorimetry, thermo gravimetric analysis, scanning electron microscope, transmission electron microscopy, and in vitro drug release and the effects were found to be satisfactory. The main problem associated with the anticancer drugs is the opsonisation which leads to the clearance of the drug by liver and spleen which was overcome by the surface modification, which helps the drug targeting to cancer cells and for overcoming the multidrug resistance [33].

Devendiran et al. [34] green synthesized gold nanoparticles containing the drug doxorubicin and the antioxidant bioflavonoid quercetin as a reducing agent with dextran sulfate as a stabilizing agent, adorned with folic acid and evaluation tests were performed for the breast cancer treatment. They have been evaluated for their in vitro physicochemical properties and biological properties and have been found to be stable. The anticancer activity was performed using the human breast cell lines MCF-7 using MTT assay, and it was proved that the formulated nanoparticles represented a potential targeted drug delivery for better cancer treatment.

Pandey et al. [35] synthesised nanoparticles of quercetin embedded with polylactic acid by novel emulsified nanoprecipitation method and evaluated its anticancer activity in human breast cancer cell lines. The physicochemical properties evaluation showed that the flavonoid release from the polymer matrix showed a sustained release and in vitro cytotoxicity studies proved the efficiency of the nanoformulation for treating cancer.

Sahu et al. [36] formulated the quercetin nanoparticles of biocompatible nature using the biocompatible and nonbiodegradable polymers like ethyl cellulose and Tween 80 by the nanoprecipitation technique for the prolonged topical delivery of the bioflavonoid for the treatment of skin cancer. The formulated nanoparticles were evaluated for their physicochemical and ex vivo skin penetration studies. From the studies, it was concluded that quercetin formulated as nanoparticles can sustain the drug release, thereby intending the decrease in the frequency and dose of the drug urging the research of quercetin novel formulations in the area of anticancer study [36]

Jalil Rashedi et al. formulated quercetin loaded chitosan nanoparticles on colon cancer induced by dextran sulfate sodium in wistar rats. The nanoparticles were formulated using the ionotropic gelation method and were characterized for all the essential criteria like physicochemical properties, in vitro drug release, reduction of microvascular density, mitosis count and measuring apoptosis percent. From the results, it was found that the quercetin loaded chitosan nanoparticles were efficient in sustaining the drug delivery and improves the site-specific delivery of the bioflavonoid [37].

\section{Fisetin}

Fisetin, known popularly as natural brown [38], is chemically $3,3^{\prime}, 4^{\prime}, 7$-tetrahydroxy flavone and a dietary flavonoid present in the foods like tomatoes, onions, mangoes, apples, strawberries, cucumbers, kiwis, nuts, and grapes [38]. Fisetin has been shown many therapeutic activities such as antioxidant activity [39], antiinflammatory activity [40, 41], antidiabetic activity [38], antiviral activity $[42,43]$, cardioprotective effect [31], and anticancer activity $[44,45]$. Fisetin was found to possess much effective therapeutic activity against cancer through various mechanisms such as inducing apoptosis of cancer cells, activation of 5' Adenosine monophosphate-activated protein kinase and acetyl-coenzyme A carboxylase [46-48] and so on. Since cancer is the dreadful disease which has gained specific attention of the researchers and fisetin a 
natural bioflavonoid in novel formulation possesses many advantages of enhanced pharmacokinetic properties, safety, economic, and specific organ targeting which can greatly help the physicians to treat cancer in a better means.

Ragelle et al. formulated fisetin a well proved antitumor agent to improve its aqueous solubility into a nanoemulsion formulation and proved its improvement in pharmacokinetic property and its antitumor therapeutic activity on intraperitoneal administration of the fisetin nanoemulsion in Lewis lung carcinoma affected mice [49].

Bothiraja et al. formulated fisetin a flavonoid with poor water solubility and the one that undergoes further metabolism in vivo, as a nano-cochelates, by trapping the technique in which the flavonoid loaded dimyristoyl-phosphatidyl choline loaded liposomal vesicles were converted into the nanochelates in the presence of $\mathrm{Ca}^{2+}$ ions This nanochelates were proved to be much stable and more effective in human breast cancer treatment [50].

\section{Genistein}

Genistein, a nutraceutical [51] soy isoflavone and a plant estrogen, a flavonoid found in soya beans and soy food, has numerous pharmacological activities such as anticholesterol activity [52], antioxidant activity, antidiabetic activity, menopausal syndrome, and anticancer activity. Although genistein is a proven isoflavone for the cervical, breast and prostrate cancer treatment, its use in this area is limited due its solubility and bioavailability, which initiates its formulation development as nanoformulation which can overcome this limitation and prove its potentiality in the treatment of cancer

Zhang et al. [53] produced flavonoid, genistein $\mathrm{d}$ - $\alpha$-tocopheryl polyethylene glycol 1000 succinate b polycaprolactone nanoparticles by modified nanoprecipitation method. This novel formulation of the flavonoid was found to be effective in improving the solubility characteristics of the antitumor flavonoid genistein and as a valuable antitumor agent in treating cervical cancer as tested in the HeLa xenograft tumour model in BALB/c nude mice.

\section{Isorhamnetin}

Isorhamnetin, a metabolite of quercetin, which is chemically Omethylated flavonol is found in plenty in blackberries, cherries, pears, and apples. The flavonoid is a highly active molecule showing a wide variety of pharmacological activities including antidiabetic, antioxidant, cardiovascular, anticancer, anti-inflammatory, hepatoprotective, and anti-obesity agents $[54,55]$. Isorhamnetin has been shown to be an effective anticancer agent for treating lung cancers [56, 57]. However, its targeting capacity is poor due to the poor aqueous solubility and bioavailability, which proves the necessity for the development of the nanoformulations for the most eligible flavonoid molecule in the treatment of cancer.

Settu and Vaiyapuri [58] studied the effect of poly lactic-co-glycolic acid isorhamnetin nanoparticles on HepG2 cancer cell lines. They concluded that the nanoformulation of isorhamnetin resulted in substantial increase in the inhibition of proliferation as compared to the plain isorhamnetin.

\section{Kaempferol}

Kaempferol, a plant nutrient widely found in many of the plant families, for example, Aspleniaceae, Aspidiaceae, Polypodiaceae, Blechnaceae, Cyatheaceae, Equisetaceae, and Ophioglossaceae and is present as a significant constituent in edible foods such as onion, mustard, turnip greens, tea, grapefruit, tea, cucumber, fennel, and peach $[59,60]$. Kaempferol based plant food intake reduces the risk of different types of cancers such as lung cancer, pancreatic cancer, ovarian malignant growth, and gastric cancer and many of the cardiovascular diseases. From various epidemiological studies, kaempferol was also proved to possess antioxidant activity, antiinflammatory activity, antidiabetic activity [61], and antimicrobial activity [62]. Although there are many pharmacological activities their usefulness is limited due to less oral bioavailability and low water solubility. Hence, researches are carried out to improve its pharmacokinetic properties; in turn, the therapeutic benefits of the flavonoid be made completely available to the suffering patients.

Luo et al. [63] studied the comparative effect of the most proven bioflavonoid kaempferol formulated as nanoparticles on the ovarian cancer cells and regular cells. The non-ionic polymers poly (ethylene oxide)-poly(propylene oxide)-poly(ethylene oxide) and poly (DLLactic acid-co-glycolic acid) were used to formulate the nanoparticles containing kaempferol. It was proved that both the polymers showed improved efficacy in decreasing the viability of cancer cells and normal cells with the PLGA kaempferol nanoparticle being superior in its specific anticancer activity [63].

Raghavan et al. [64] synthesized gold nanoparticles contain the flavonol, kaempferol, and proved their cell toxic effect on the MCF-7 cancer cell line. The results of the study showed that the nanoparticles were found to be successful by reducing the vivacity of MCF-7 breast cancer cells as confirmed by the ethidium bromideacridine orange staining method and urged programmed cell death as confirmed by the CyQuant assay method [64].

\section{Luteolin}

Luteolin, a flavone found in foods which we intake which includes, cabbage, carrots, apple skin, green pepper, and chamomile tea. It was also studied for various therapeutical activities such as antitumorigenic [65], anti-inflammatory, antidiabetic, and antioxidant [38]. Luteolin requires high bioavailability to exhibit its pharmacological activity in vivo, and due to its low bioavailability in vivo by the oral route, the novel formulation development may prove to be helpful to achieve its maximum pharmacological activity.

Majumdar et al. studied the effect of the bioflavonoid luteolin in nanoparticle formulation to improve its aqueous solubility, bioavailability, and anticancer efficacy of the flavonoid. The PLAPEG-OMe polymer was used for formulating luteolin nanoparticles and the evaluation of the formulated nanoparticles for lung, neck, and head anticancer activity was done by in vitro and in vivo methods. The various cell lines such as $\mathrm{H} 292$ and Tu212 were used for the study, which showed the inhibitory activity of the nanoparticles against the cancer cell lines and the in vivo anticancer activity by tumour xenograft mouse model which also proved a significant inhibitory effect on the tumour cells [66].

\section{Hesperetin}

Hesperidin, a flavone glycoside, an abundant plant constituent present in limes and lemons, was studied for many pharmacological effects, such as antidiabetic, antioxidant, anticancer, antiinflammatory effects, and so on. It was found to prevent cell death. Although it possesses many pharmacological activities, its use in the medicinal field is limited due to its limited aqueous solubility and bioavailability, which can be rectified to a certain extent by formulating the flavonoid as nanoformulation which can also serve as a measure to target the flavonoid to cancer cells.

Gurushankar et al. formulated, characterized, and evaluated the bioflavonoid hesperetin nanoparticles produced using the polymers Eudragit E 100 by the nanoprecipitation technique for its efficiency in treating oral cancer. The results concluded that flavonoid nanoparticles were effective in dose-dependent toxic effects on the cancer cells, changes in the capacity of mitochondrial membrane, improved intracellular reactive oxygen species, necrobiotic morphological changes, and also DNA damage, which urges its development as novel particles for the treatment of dreadful diseases [67].

\section{Naringenin}

Naringenin, chemically a flavanone glycoside is $\left(4^{\prime}, 5,7-\right.$ trihydroxyflavanone) found to be a significant constituent in various citrus fruits, tomatoes [68], cherries [69], and cocoa is found to be the potent anticancer agent in treating liver and gastric cancer $[70,71]$. It was also studied for its antioxidant, liver protective, antiinflammatory, cardiovascular, gastrointestinal, and immunity-boosting effects (72-77). Naringenin was found to increase the therapeutic activity of anticancer drugs like doxorubicin, and the drug seems to 
possess cardiotoxicity on long-term usage. Naringenin seems to possess low bioavailability and solubility, which makes it's clear to make modifications in formulations for increasing its pharmacokinetic parameters and also to make the flavonoid disease-specific.

Winarti et al. have studied the in vitro anticancer activity of the flavonoid naringenin nanoparticles formulated using the chitosan polymer. T47D breast cancer cell line was used for the research and was found that the encapsulated naringenin showed an increased cell toxic effect. Also, it resulted in the increased cellular uptake and caused induction in the programmed cell death [78].

Parashar et al. [79] studied the naringenin loaded in polycaprolactone polymer as nanoparticles linked with the hyaluronic acid to increase the target specificity of the flavonoid, which is a potential drug candidate in the treatment of lung cancer The results revealed that the flavonoid naringenin is a potential candidate for lung cancer treatment, as proved by the cell toxicity studies on A549 cells and J774 cells.

\section{Rutin}

Rutin, a bioflavonoid found in buckwheat bran, citrus fruits, black tea, clove, rose, rue possesses many of the therapeutic activities including the antioxidant activity [80], cardioprotective activity, anti-atherosclerotic [33], anticancer activities [81], and many other useful pharmacological properties. Although it has various pharmacological properties, its exploration in the field of medicine is much reduced due to the low solubility and bioavailability, which can be overcome by making modifications in the formulation using various polymers which are of a degradable type and producing the nanoformulations [82]

Asfour and Mohsen [83] formulated and studied the effect of the less soluble bioflavonoid rutin as nanospheres using the polymer Eudragit S 100 with various concentration of stabiliser poloxamer 188 , to target the cancerous cells in the colon region of the body. They proved the rutin nanospheres efficiency in the treatment of colonic cancer as showed by the two-fold increased activity of the nanoformulation in the cell toxic effect of the bioflavonoid rutin [83].

Ahmad et al. [84] developed a nanoemulsion formulation of the bioflavonoid rutin and evaluated for its anticancer activity on the prostatic cancer cell lines $\mathrm{PC}-3$, proving its efficiency in treating prostatic cancer.

\section{CONCLUSION}

Bioflavonoids may have the potential to help with cardiac well being cancer prevention, and different issues associated with oxidative stress and inflammation, such as allergies and asthma. They are also readily available in a healthy diet. The fruits, vegetables, and other foods rich in flavonoids are high in fibre, nutrients and minerals. They are also low in saturated fats and cholesterol, making them healthy choices for your general well-being. Many of the daily foods can be used as drugs. Among them, the flavonoids which find their source in many of the daily foods is proved through many experiments to possess potential therapeutic activities in treating many of the diseases. Cancer is considered to be the disease which is of major concern worldwide. Due to the various side effects in using the conventional system of medicine, modern developments have been in the full wing in using these plant-derived constituents, especially the flavonoids for the formulation as novel drug delivery systems such as nanoparticles, nanoemulsions, and liposomes which will be a boon to the human society in treating cancer.

\section{FUNDING}

Nil

\section{AUTHORS CONTRIBUTIONS}

All authors contributed equally.

\section{CONFLICT OF INTERESTS}

The author declared that there is no conflict of interest.

\section{REFERENCES}

1. Atanasov AG, Waltenberger B, Pferschy Wenzig EM, Linder T, Wawrosch C, Uhrin $\mathrm{P}$, et al. Discovery and resupply of pharmacologically active plant-derived natural products: a review. Biotechnol Adv 2015;33:1582-614.

2. Gurnani N, Mehta D, Gupta M, Mehta BK. Natural products: source of potential drugs. Afr J Basic Appl Sci 2014;6:171-86.

3. Leopoldini M, Russo N, Chiodo S, Toscano M. Iron chelation by the powerful antioxidant flavonoid quercetin. J Agric Food Chem 2006;54:6343-51.

4. Pawar HA. Natural products as a source of lead to the design of new drugs. Nat Prod Chem Res 2014;2:156

5. Lu J, Papp LV, Fang J, Rodriguez Nieto S, Zhivotovsky B, Holmgren A, et al. Inhibition of mammalian thioredoxin reductase by some flavonoids: Implications for myricetin and quercetin anticancer activity. Cancer Res 2006;66:4410-8.

6. Bukhari SB, Memon S, Mahroof Tahir M, Bhanger MI. Synthesis, characterization and antioxidant activity copper-quercetin complex. Spectrochim Acta A Mol Biomol Spectrosc 2009;71:1901-6.

7. Khnau J. The flavonoids a class of semi-essential food components: their role in human nutrition. World Rev Nutr Diet 1976;24:117-91.

8. Kumar S, Pandey AK. Antioxidant, lipo-protective and antibacterial activities of phytoconstituents present in Solanum xanthocarpum root. Int Rev Biophys Chem 2012;3:42-7.

9. Hertog MG, Kromhout D, Aravanis C. Flavonoid intake and long term risk of coronary heart disease and cancer in seven countries study. Arch Int Med 1995;155:381-6.

10. Ferrndiz ML, Nair AG, Alcaraz MJ. Inhibition of sheep platelet arachidonate metabolism by flavonoids from spanish and Indian medicinal herbs. Pharmazie 1990;45:206-8.

11. Ferrndiz ML, Alcaraz MJ. Anti-inflammatory activity and inhibition of arachidonic acid metabolism by flavonoids. Agents Actions 1991;32:283-8.

12. Stefani ED, Boffetta P, Deneo Pellegrini H, Mendilaharsu M, Carzoglio JC, Ronco A, et al. Dietary antioxidants and lung cancer risk: a case-control study in Uruguay. Nutr Cancer 1999;34:100-10.

13. Alcaraz MJ, Ferrandiz ML. Modification of arachidonic metabolism by flavonoids. J Ethnopharmacol 1987;21:209-29.

14. Fotsis T, Pepper MS, Aktas E, Breit S, Rasku S, Adlercreutz H, et al. Flavonoids, dietary-derived inhibitors of cell proliferation and in vitro angiogenesis. Cancer Res 1997;57:2916-21.

15. Laughton MJ, Evans PJ, Moroney MA, Hoult JR, Halliwell B. Inhibition of mammalian 5-lipoxygenase and cyclo-oxygenase by flavonoids and phenolic dietary additives. Relationship to antioxidant activity and to iron ion-reducing ability. Biochem Pharmacol 1991;42:1673-81.

16. Al Rashid MH, Deepak Bharadwaj PVP, Majumder S, Mandal V, Pal M, Mandal SC, et al. Antioxidant and anticancer activity of extract and fractions obtained from diospyros melanoxylon roxb. Leaves and correlation with their polyphenolic profiles. Int J Pharm Pharm Sci 2018;10:6-16.

17. Madhu CS, Sharada AC. Evaluation of heamagglutination and anti-cancer potential from indian dietary plants. Int J Pharm Pharm Sci 2018;10:105-8.

18. Neerugatti DB, GR Battu, R Bandla. Cytotoxicity activity of some Indian medicinal plants. Int J Curr Pharm Res 2016;8:57-9.

19. Devi KP, Balan DJ. Flavonoids and their anti-cancer effect is targeting the major signalling pathways involved. In: Recent advances in the molecular mechanism of flavonoids. Houston, Texas: Studium Press (India Pvt. Ltd.); 2018. p. 1-22.

20. Caltagirone S, Rossi C, Poggi A, Ranelletti FO, Natali PG, Brunetti $\mathrm{M}$, et al. Flavonoids apigenin and quercetin inhibit melanoma growth and metastatic potential. Int J Cancer 2000;87:595-600.

21. Hegarty VM, May HM, Khaw KT. Tea drinking and bone mineral density in older women. Am J Clin Nutr 2000;71:1003-7.

22. Di Carlo G, Autore G, Izzo AA, Maiolino P, Mascolo N, Viola P, et al. Inhibition of intestinal motility and secretion by flavonoids 
in mice and rats: structure-activity relationships. J Pharm Pharmacol 1993;45:1054-9.

23. Wang HK, Xia Y, Yang ZY, Natschke SL, Lee KH. Recent advances in the discovery and development of flavonoids and their analogues as antitumor and anti-HIV agents. Adv Exp Med Biol 1998;439:191-225.

24. Wild C, Fasel J. Effect of a flavonoid on the capillary resistance of the rectal mucosa in hepatic cirrhosis. Am J Proctol 1969;20:60-3.

25. Ravishankar D, Rajora AK, Greco F, Osborn HM. Flavonoids as prospective compounds for anti-cancer therapy. Int J Biochem Cell Biol 2013;45:2821-31

26. Li S, Pan MH, Lai CS, Lo CY, Dushenkov S, Ho CT. Isolation and syntheses of poly-methoxyflavones and hydroxylated polymethoxyflavones as inhibitors ofHL-60 cell lines. Bioorg Med Chem 2007;15:3381-9.

27. Kawaii S, Tomono Y, Katase E, Ogawa K, Yano M. Antiproliferative activity of flavonoids on several cancer cell lines. Biosci Biotechnol Biochem 1999;63:896-9.

28. Saewan N, Koysomboon S, Chantrapromma K. Anti-tyrosinase and anti-cancer activities of flavonoids from Blumea balsamifera DC. J Med Plants Res 2011;5:1018-25.

29. Zhou JR, Mukherjee P, Gugger ET, Tanaka T, Blackburn GL, Clinton SK, et al. Inhibition of murine bladder tumorigenesis by soy isoflavones via alterations in the cell cycle, apoptosis, and angiogenesis. Cancer Res 1998;58:5231-8.

30. Veeramuthu D, Raja WR, Al-Dhabi WA, Savarimuthu I. Flavonoids: anticancer properties. In: Flavonoids-From biosynthesis to human health; 2017. p. 287-303.

31. Saravanan D, Maheswaran A, Padmavathy J, Angel P. Molecular mechanism of flavonoids on cardiac diseases. In: Devi PK. editors. Recent advances in the molecular mechanism of flavonoids. Houston, TX: Studium Press (India Pvt. Ltd.); 2018. p. 183-201.

32. Bhagwat RR, Vaidhya IS. Novel drug delivery systems: an overview. Int J Pharm Sci Res 2013;4:970-82.

33. Saha C, Kaushik A, Das A, Pal S, Majumder D. Anthracycline drugs on modified surface of quercetin-loaded polymer nanoparticles: a dual drug delivery model for cancer treatment. PLoS One 2016;11:e0155710.

34. Devendiran RM, Chinnaiyan SK, Yadav NK, Ramanathan G, Singaravelu S, Perumal SP, et al. Facile synthesis and evaluation of quercetin reduced and dextran sulphate stabilized gold nanoparticles decorated with folic acid for active targeting against breast cancer. RSC Adv 2016;4:1-14.

35. Pandey SK, Patel DK, Thakur R, Mishra DP, Maiti P, Haldar C, et al. Anti-cancer evaluation of quercetin embedded PLA nanoparticles synthesized by emulsified nanoprecipitation. Int J Biol Macromol 2015;75:521-9.

36. Sahu S, Saraf S, Kaur CD, Saraf S. Biocompatible nanoparticles for sustained topical delivery of anticancer phytoconstituent quercetin. Pak J Biol Sci 2013;16:601-9.

37. Jalil Rashedi, Amir Ghorbani Haghjo, Mehran Mesgari Abbasi, Ali Dastranj Tabrizi, Shabdi Yaquobi, Davoud Sanajou, et al. Anti-tumor effect of quercetin loaded chitosan nanoparticles on induced colon cancer in wistar rats. Adv Pharm Bull 2019;9:409-15.

38. Shivsharan B, Baburao D, Chandakavathe N. Anti-diabetic potential of Flavonoids and their molecular mechanisms. In: Devi KP. editor. Recent Advances in the Molecular Mechanism of Flavonoids. Houston, Texas: Studium Press (India Pvt. Ltd.); 2018. p. 104-39.

39. Naeimi AF, Alizadeh M. Antioxidant properties of the flavonoid fisetin: An updated review of in vivo and in vitro studies. Trends Food Sci Tech 2017;70:34-44.

40. Wang L, Tu YC, Lian TW, Hung JT, Yen JH, Wu MJ, et al. Distinctive antioxidant and anti-inflammatory effects of flavonols. J Agric Food Chem 2006;54:9798-804.

41. Hendriks JJ, de Vries HE, van der Pol SM, van den Berg TK, van Tol EA, Dijkstra CD, et al. Flavonoids inhibit myelin phagocytosis by macrophages; a structure-activity relationship study. Biochem Pharmacol 2003;65:877-85.
42. Inouye Y, Yamaguchi K, Take Y, Nakamura S. Inhibition of avian myeloblastosis virus reverse transcriptase by flavones and isoflavones. J Antibiot (Tokyo) 1989;42:1523-5.

43. Chu SC, Hsieh YS, Lin JY. Evaluation of the anti-herpetic activity of methanol seed extract and fractions of securigera securidaca in vitro. J Nat Prod 2013;55:179-83.

44. Lall RK, Adhami VM, Mukhtar H. Dietary flavonoid fisetin for cancer prevention and treatment. Mol Nutr Food Res 2016;60:1396-405.

45. Pal HC, Pearlman RL, Afaq F. Fisetin and its role in chronic diseases. Adv Exp Med Biol 2016;928:213-44.

46. Zhang WB, Wang Z, Shu F, Jin YH, Liu HY, Wang QJ, et al. Activation of AMP-activated protein kinase by temozolomide contributes to apoptosis in glioblastoma cells via p53 activation and mTORC1 inhibition. J Biol Chem 2010;285:40461-71.

47. Kim HJ, Kim SK, Kim BS, Lee SH, Park YS, Park BK, et al. Apoptotic effect of quercetin on HT-29 colon cancer cells via the AMPK signaling pathway. J Agric Food Chem 2010;58:8643-50.

48. Kim DY, Park MW, Yuan HD, Lee HJ, Kim SH, Chung SH, et al. Compound $\mathrm{K}$ induces apoptosis via CAMK-IV/AMPK pathways in HT-29 colon cancer cells. J Agric Food Chem 2009;57:10573-8.

49. Ragelle H, Crauste Manciet S, Seguin J, Brossard D, Scherman D, Arnaud $\mathrm{P}$, et al. Nanoemulsion formulation of fisetin improves bioavailability and antitumour activity in mice. Int J Pharm 2012;427:452-9.

50. Bothiraja C, Yojana BD, Pawar AP, Shaikh KS, Thorat UH Fisetin-loaded nanocochleates: formulation, characterisation, in vitro anticancer testing, bioavailability and biodistribution study. Expert Opin Drug Delivery 2014;11:17-29.

51. Meghwal M, Sahu CK. Soy isoflavonoids as a nutraceutical for human health: an update. J Cell Sci Ther 2015;6:1000194.

52. Suthar AC, Banavalikar MM, Biyani MK. Pharmacological activities of genistein, an isoflavone from soy (Glycine max): Part II-anti-cholesterol activity, effects on osteoporosis and menopausal symptoms. Indian J Exp Biol 2001;39:520-5.

53. Zhang H, Liu G, Zeng X, Wu Y, Yang C, Mei L, et al. Fabrication of genistein-loaded biodegradable TPGS-b-PCL nanoparticles for improved therapeutic effects in cervical cancer cells. Int J Nanomed 2015;10:2461-73.

54. Kumar SK, Manju V. Pharmacological applications of isrhamnetin: a short review. Int J Trend Sci Res Dev 2017:1:672-8.

55. Ma G, Yang C, Qu Y, Wei H, Zhang T, Zhang $\mathrm{N}$, et al. The flavonoid component isorhamnetin in vitro inhibits proliferation and induces apoptosis in eca-109 cells. Chem Biol Interact 2007;167:153-60.

56. Lee K, Kwon M, Baek I, Kim H, Lee H, Park H, et al. Ant proliferation effects of isorhamnetin isolated from Persicaria thunbergii on cancer cell lines. Nat Prod Sci 2006;12:214-26.

57. Steffen Y, Gruber C, Schewe T, Sies H. Mono-O-methylated flavanols and other flavonoids as inhibitors of endothelial NADPH oxidase. Arch Biochem Biophys 2008;469:209-19.

58. Settu K, Vaiyapuri M. Formulation and evaluation of isorhamnetin loaded poly lactic-co-glycolic acid nanoparticles. Asian J Pharm Clin Res 2017;10:177-81.

59. Yang RY, Lin S, Kuo G. Content and distribution of flavonoids among 91 edible plant species. Asia Pac J Clin Nutr 2008;17 Suppl 1:275-9.

60. Franke AA, Custer LJ, Arakaki C, Murphy SP. Vitamin C and flavonoid levels of fruits and vegetables consumed in Hawaii. Food Compost Anal 2004;17:1-35.

61. Andrade Cetto A, Wiedenfeld H, Revilla MC, Sergio IA. Hypoglycemic effect of equisetum myriochaetum aerial parts on streptozotocin-diabetic rats. J Ethnopharmacol 2000;72:129-33

62. J M Calderon Montano, E Burgos Moron, C Perez Guerrero, M Lopez Lazaro. A review on the dietary flavonoid kaempferol. Mini Rev Med Chem 2011;11:298-344.

63. Luo H, Jiang B, Li B, Li Z, Jiang BH, Chen YC, et al. Kaempferol nanoparticles achieve strong and selective inhibition of ovarian cancer cell viability. Int J Nanomed 2012;7:3951-9. 
64. Raghavan BS, Kondath S, Anantanarayanan R, Rajaram R. Kaempferol mediated synthesis of gold nanoparticles and their cytotoxic effects on MCF-7 cancer cell line. Process Biochem 2015;50:1966-76.

65. Chahar MK, Sharma N, Dobhal MP, Joshi YC. Flavonoids: a versatile source of anticancer drugs. Pharmacogn Rev 2011;5:1-2.

66. Majumdar D, Jung KH, Zhang H, Nannapaneni S, Wang X, Amin AR, et al. Luteolin nanoparticle in chemoprevention: in vitro and in vivo anticancer activity. Cancer Prev Res (Phila) 2014;7:65-73.

67. Gurushankar K, Gohulkumar M, Prasad NR, Krishnakumar N. Synthesis, characterization and in vitro anticancer evaluation of hesperetin-loaded nanoparticles in human oral carcinoma(KB) cells. Adv Nat Sci Nanosci Nanotechnol 2014;5:15006.

68. Le Gall G, DuPont MS, Mellon FA, Davis AL, Collins G], Verhoeyen ME, et al. Characterization and content of flavonoid glycosides in genetically modified tomato (Lycopersicon esculentum) fruits. J Agric Food Chem 2003;51:2438-46.

69. Wang H, Nair MG, Strasburg GM, Booren AM, Gray JI. Antioxidant polyphenols from tart cherries (Prunus cerasus). J Agric Food Chem 1999;47:840-4.

70. Chang H, Mi M, Ling W, Zhu J, Zhang Q, Wei N, et al. Structurally related cytotoxic effects of flavonoids on human cancer cells in vitro. Arch Pharm Res 2008;31:1137-44.

71. Kanno S, Tomizawa A, Hiura T, Osanai Y, Shouji A, Ujibe M, et al. Inhibitory effects of naringenin on tumor growth in human cancer cell lines and sarcoma S-180-implanted mice. Biol Pharm Bull 2005;28:527-30.

72. Kim JH, Lee JK. Naringenin enhances NK cell lysis activity by increasing the expression of NKG2D ligands on burkitt's lymphoma cells. Arch Pharm Res 2015;38:2042-8.

73. Lee YS, Reidenberg MM. A method for measuring naringenin in biological fluids and its disposition from grapefruit juice by man. Pharmacology 1998;56:314-7.

74. Middleton E Jr, Kandaswami C. Effects of flavonoids on immune and inflammatory cell functions. Biochem Pharmacol 1992;43:1167-79.
75. Rajadurai M, Prince PS. Naringin ameliorates mitochondrial lipid peroxides, antioxidants and lipids in isoproterenolinduced myocardial infarction in wistar rats. Phytother Res 2009;23:358-62.

76. Rimm EB, Katan MB, Ascherio A, Stampfer MJ, Willett WC. Relation between intake of flavonoids and risk for coronary heart disease in male health professionals. Ann Intern Med 1996;125:384-9.

77. Al-Rejaie SS, Abuohashish HM, Al-Enazi MM, Al-Assaf $\mathrm{AH}$ Parmar MY, Ahmed MM, et al. Protective effect of naringenin on acetic acid-induced ulcerative colitis in rats. World J Gastroenterol 2013;19:5633-44

78. Winarti L, Sari LO, Nugroho AE. Naringenin loaded chitosan nanoparticles formulation and it's in vitro evaluation against T47D breast cancer cell line. Indones J Pharm 2015;26:147-57.

79. Parashar P, Rathor M, Dwivedi M, Saraf SA. Hyaluronic acid decorated naringenin nanoparticles: appraisal of the chemopreventive and curative potential for lung cancer. Pharmaceutics 2018;10:E33.

80. Kamalakkannan N, Prince PS. Antihyperglycaemic and antioxidant effect of rutin, a polyphenolic flavonoid, in streptozotocin-induced diabetic wistar rats. Basic Clin Pharmacol Toxicol 2006;98:97-103.

81. Luo H, Jiang BH, King SM, Chen YC. Inhibition of cell growth and VEGF expression in ovarian cancer cells by flavonoids. Nutr Cancer 2008;60:800-9.

82. Padmavathy J, Sathesh Kumar S. Bioflavonoids with anticancer activity and their novel formulations. Asian J Pharm Clin Res 2018;11:1-6.

83. Asfour MH, Mohsen AM. Formulation and evaluation of $\mathrm{pH}-$ sensitive rutin nanospheres against colon carcinoma using HCT-116 cell line. J Adv Res 2018;9:17-26.

84. Ahmad M, Sahabjada AS, Akhtar J, Hussain A, Badaruddeen M, Arshad M, et al. Development of a new rutin nanoemulsion and its application on prostate carcinoma PC3 cell line. EXCLI J 2017;16:810-23 